\title{
Correlation between Neutrophil Gelatinase-associated Lipocalin and highly sensitive $C$ reactive protein as markers for early detection of acute kidney injury in neonatal sepsis.
}

\author{
Moftah Mohamed Rabeea ${ }^{1}$, Mohamed Abdel- Karim Mohamed ${ }^{1}$ \\ Ibrahim Metwally Bioumy ${ }^{2}$, El Sayed Shaaban Abdel- Aziz ${ }^{1}$ \\ 1- Pediatrics departments Faculty of Medicine- Al-Azhar University. \\ 2- Clinical pathology Departments Faculty of Medicine- Al-Azhar University.
}

\begin{abstract}
Introduction

Sepsis is the commonest cause of neonatal mortality and is responsible for 30-50\% of total neonatal deaths each year in developing countries. Neonatal sepsis is one of the commonest causes of Acute Kidney Injury (AKI) in newborns and it is associated with poor outcomes.
\end{abstract}

\section{Aim of the study}

To investigate the value of Neutrophil Gelatinase-associated Lipocalin and highly sensitive C reactive protein as markers for early detection of acute kidney injury in neonatal sepsis.

\section{Patients and Methods}

This prospective study was conducted on 50 neonates admitted to the Neonatal Intensive Care Unit with neonatal sepsis at our university hospital (GI) and 30 apparently healthy neonates matched for gestational age and weight as a control group (GII). The septic group (GI) included all neonates (age 5-28 days) with neonatal sepsis and were classified into two subgroups according to the presence of AKI. After detailed history and complete clinical examination, all cases were subjected to the following investigations: $\mathrm{CBC}, \mathrm{ABG}$, blood culture, serum electrolytes and creatinine, CRP, hs-CRP and NGAL.

\section{Results}

Only 64\% of all septic cases showed positive qualitative CRP (> 6mg/L) while $86 \%$ of them showed positive hs-CRP of ( $>0.5 \mathrm{mg} / \mathrm{L}$ ) with highly significant differences $(\mathrm{p}<0.001)$. Blood culture was positive only in $52 \%$ of cases. In this study, hs-CRP was significantly higher in septic neonates with AKI (27 cases) than those without AKI (16 cases) (p < 0.001). NGAL level was significantly higher in septic neonates than the control group $(p<0.001)$. Also NGAL level was markedly elevated in septic cases with AKI compared to non AKI subgroup with highly significant differences $(\mathrm{p}<0.001)$. There was a positive correlation between NGAL and hs-CRP in septic cases with AKI.

\section{Conclusion}

Hs-CRP was proved to be very useful biochemical marker for early detection of neonatal sepsis compared to qualitative CRP. Serum NGAL and hs-CRP can be considered as very useful biochemical markers for early detection of AKI in septic neonates before any rise in serum creatinine.

\section{Keywords}

Acute kidney injury, highly sensitive $\mathrm{C}$ reactive protein, neonatal sepsis, Neutrophil gelatinase associated lipocalin.

Running Title Early detection of acute kidney injury in neonatal sepsis

\section{Correspondence El Sayed Shaaban Abdel -Aziz}

Faculty of Medicine- Al-Azhar University, Candidate for fulfillment of Master Degree in pediatrics.

Email: drsaid43cc@gmaill.com , Mobile: 01117334615

\section{geget: The Journal of the Egyptian Society of Pediatric Nephrology and Transplantation (ESPNT) geget https://geget.journals.ekb.eg/ \\ Published by ESPNT http://espnt.net/ \\ Cohosted by Egyptian Knowledge Bank https://www.ekb.eg}




\section{Introduction}

Neonatal sepsis, is a constellation of clinical and laboratory findings associated with invasive infection during the first 28 days of life. IT is an important cause of mortality for neonates and remains a clinical challenge especially for asymptomatic infants. Neonates with SIRS have a spectrum of clinical symptoms that represent progressive stages of the pathologic process [1].

Critically ill neonates are at greater risk of having AKI as they are commonly exposed to nephrotoxic medications and have frequent infections, which lead to multiorgan failure [2]. Acute kidney injury is classically defined as a sudden decline in kidney function resulting in derangements in fluid balance and electrolytes. Highly sensitive C-reactive protein might be helpful as a predictive factor of acute kidney injury in many situations beside its value as a sensitive sepsis marker [3]. The difference between CRP and hs-CRP is contained in the "hs" abbreviation: "highly sensitive". CRP traditionally measured down to concentrations of $3-5 \mathrm{mg} / \mathrm{L}$, whereas through changes in detection systems, hs-CRP measures down to concentrations around $0.04 \mathrm{mg} / \mathrm{L}[1]$.

Neutrophil Gelatinase associated Lipocalin (NGAL) is a 198 amino acid glycoprotein encoded by a gene located on the chromosome locus $3 p 11$. It was first isolated from mouse kidney cells infected with a simian virus (SV-40). The expression of NGAL rises 1000-fold in humans and rodents in response to renal tubular injury, and it appears so rapidly in the urine and serum that it is useful as an early biomarker of renal injury [4].

\section{Aim of the study}

The aim of this work is to estimate Neutrophil Gelatinase associated Lipocalin (NGAL) and highly sensitive $\mathrm{C}$ reactive protein (hs-CRP) as markers for early detection of acute kidney injury $(\mathrm{AKI})$ in neonatal sepsis.

\section{Patients and Methods}

This prospective case-control study was conducted on 50 neonates admitted to the Neonatal Intensive Care Unit (NICU) with neonatal sepsis at our university hospital and 30 apparently healthy neonates matched for gestational age and sex a control group. This study was carried out during the period from (June 2018 to June 2019).

Sample size:

$$
n=\frac{N}{N(e)^{2}}
$$

Where $\mathrm{n}$ is the sample size, $\mathrm{N}$ is the population size, and $\mathrm{e}$ is the level of precision.

\section{** Inclusion criteria}

Neonates with sepsis (age 5-28 days) based on clinical, hematological findings and / or positive blood culture. Neonatal AKI was defined by serum creatinine level, serum NGAL and hs-CRP.

Neonates with antenatally diagnosed renal abnormality, multiple congenital anomalies, inborn error of metabolism, Hypoxic ischemic encephalopathy were excluded.
- $\quad$ Septic neonates (GI) were classified into: Group IA: Newborns with neonatal sepsis and acute kidney injury

$(\mathrm{n}=27)$ and Group IB: Newborns with neonatal sepsis and no acute kidney injury $(\mathrm{n}=23)$. While the Control group (Group II = 30): Thirty healthy neonates from unknown matching with the cases as regard to gestational age, sex and weight were included as a control group.

Ethical Aspect: The approval for this study was obtained from the ethical committee of the Pediatric Department of our University. Informed written consent was obtained from parents of included neonates after explanation of the aim of the study and its benefits for newborns.

All neonates were subjected to the following

Full history: Prenatal history (maternal fever, maternal UTI, drugs ...etc.), Obstetric history (mode of delivery, gestational age, obstetric complications to the baby or the mother), Natal history (premature rupture of membrane, maternal fever, etc. ) and Post natal history included most common symptoms of sepsis (vomiting, poor feeding, failure to thrive, not doing well, fever, jaundice, convulsion...etc.).

Clinical examination: Gestational age: using Ballard score, Anthropometric measurements (weigh, length, head circumference) and Apgar score at 1 and 5 minutes.

Detection of clinical signs of sepsis e.g.: Respiratory dysfunction (apnea, signs of respiratory distress), Cardiovascular dysfunction (tachycardia, hypotension, prolonged capillary refill time, shock), GIT dysfunction (abdominal distension, feeding intolerance, poor suckling, hepatosplenomegally, jaundice, ileus) and Neurological dysfunction (irritability, Hypotonia, disturbed consciousness and convulsion).

Laboratory investigations: A serum separator tube (SST) was used, $5 \mathrm{ml}$ blood Samples were collected and allowed to clot for 30 minutes before centrifugation for 15 minutes at $1000 \mathrm{x} \mathrm{g}$. Serum was removed immediately and samples were stored at $\leq$ $-20^{\circ} \mathrm{C}$. Repeated freeze-thaw cycles were avoided.

1. CRP (positive $>6 \mathrm{mg} / \mathrm{l}$ ): Using qualitative latex agglutination test.

2. Serum electrolytes $(\mathrm{Na}+, \mathrm{K}+$ and $\mathrm{Ca}++)$.

3. Serum creatinine (two samples were taken from septic neonates with age range 5-28 days). The duration between first (on admission) and second sample (three days after admission) for observation any rising in serum creatinine). For diagnosis of AKI in neonates serum creatinine $(>0.6 \mathrm{mg} / \mathrm{dl})$ is diagnostic [5]. 4. Highly sensitive CRP (hs-CRP: using ELISA) (positive $>0.5$ $\mathrm{mg} / \mathrm{l})$

5. Measurement of serum NGAL levels: using ELISA (positive $>144 \mathrm{ng} / \mathrm{ml})[4]$.

\section{Statistical analysis}

Data were collected, coded, revised and entered to the statistical package for social science (SPSS) version 20. Qualitative data were presented as number and percentages while quantitative data were presented as mean, standard deviation and range. Pearson correlation coefficient were used to assess the relation between two parameters in the same group. $P$ value below 0.05 was considered significant. 


\section{Results}

Creatinine levels, BUN and serum potassium levels were significantly higher in cases group as compared to control group $(\mathrm{P}<0.001)$ (Table 1). On the other hand this table shows that serum sodium levels, serum Calcium levels, blood glucose levels, HB\% levels, and platelet count were significantly lower among neonatal sepsis group as compared to control group ( $\mathrm{P}$ $<0.05$ ) (Table 1).

Cases only were significantly associated with higher hsCRP, NGAL, CRP and positive blood culture (Table 2).
Levels of serum NGAL (sNGAL) and hs-CRP were significantly higher $(\mathrm{p}<0.001)$ in the -AKI subgroup as compared to no-AKI subgroup (Table 3 ). There was a positive correlation between NGAL and hs-CRP in septic cases with AKI $(r=0.33)$ (Figure 1).

The ROC curve shows that Hs-CRP can be used to discriminate between AKI patients and non-AKI patients at a cutoff level of $>3.5$, with $88.5 \%$ sensitivity, $95.6 \%$ specificity, 95.26\% PPV and 89.26\% NPV (Figure 2). The ROC curve shows that serum NGAL can predict acute kidney injury (AKI) at cutoff point of $(144 \mathrm{ng} / \mathrm{ml})$ with Sensitivity of $100 \%$ and Specificity of $95.6 \%$ and accuracy of $98 \%$ (Figure 3 ).

Table 1 Comparison of biochemical \& hematological parameters between cases and controls.

\begin{tabular}{|c|c|c|c|}
\hline & Controls $(n=30)$ & Cases $(\mathbf{n}=\mathbf{5 0})$ & $P$ value \\
\hline $\begin{array}{c}\text { Serum creatinine }(\mathrm{mg} / \mathrm{dl}) \\
\left.1^{\text {st }} \text { sample(5-25days }\right) \\
2^{\text {nd }} \text { sample }(8-28 d a y s)\end{array}$ & $\begin{array}{c}0.3 \pm 0.1 \\
0.2 \pm 0.14\end{array}$ & $\begin{array}{l}0.4 \pm 0.17 \\
1.0 \pm 0.67\end{array}$ & $\begin{array}{l}<0.001 \\
<0.001\end{array}$ \\
\hline Blood Urea Nitrogen (mg/dl) & $9.4 \pm 1.8$ & $71.83 \pm 17.9$ & $<0.001$ \\
\hline Blood pH & $7.38 \pm 0.03$ & $7.3 \pm 0.15$ & 0.051 \\
\hline Base deficit (mg/dl) & $5.23 \pm 1$ & $5.16 \pm 1.24$ & 0.8 \\
\hline Serum $\mathrm{Na}^{+}(\mathbf{m g} / \mathbf{d l})$ & $137.5 \pm 3.2$ & $127.3 \pm 4.7$ & $<0.001$ \\
\hline Serum K $\mathrm{K}^{+}$(mg/dl) & $3.9 \pm 0.31$ & $4.5 \pm 0.6$ & $<0.001$ \\
\hline Serum Ca $^{++}(\mathrm{mg} / \mathrm{dl})$ & $9.32 \pm 0.5$ & $6.8 \pm 0.6$ & $<0.001$ \\
\hline Blood Glucose (mg/dl) & $57.3 \pm 3.9$ & $33.95 \pm 6.7$ & $<0.001$ \\
\hline Heamoglobin \% (g \%) & $15.88 \pm 1.79$ & $14 \pm 1.9$ & 0.002 \\
\hline Red Blood Cell $\left(\times 10^{6} / \mathrm{mm}^{3}\right)$ & $5 \pm 0.38$ & $4.86 \pm 0.4$ & 0.141 \\
\hline 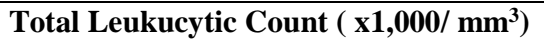 & $12.6 \pm 1.47$ & $30 \pm 5.3$ & 0.063 \\
\hline Platelets $\left(\mathrm{x}^{2} 0^{3} / \mathrm{mm}^{3}\right)$ & $232.25 \pm 68.3$ & $64.88 \pm 41$ & $<0.001$ \\
\hline
\end{tabular}

Table 2 Comparison between cases \& control group regarding laboratory investigations

\begin{tabular}{|c|c|c|c|c|c|}
\hline \multicolumn{2}{|c|}{ Item } & \multirow{2}{*}{$\begin{array}{c}\text { Cases } \\
\text { No }=\mathbf{5 0} \\
43(86) \%\end{array}$} & \multirow{2}{*}{$\begin{array}{c}\begin{array}{c}\text { Control } \\
\text { No }=30\end{array} \\
0(0) \%\end{array}$} & $\mathbf{X}_{2}$ & $\mathbf{P}$ \\
\hline hsCRP & Positive & & & \multirow{2}{*}{14.1} & \multirow{2}{*}{$<0.001$} \\
\hline (No \%) & Negative & $7(14 \%)$ & $30(100 \%)$ & & \\
\hline sNGAL (ng/ml) & Positive & $27(54 \%)$ & $1(3) \%$ & 10.3 & $<0.001$ \\
\hline \multirow{2}{*}{$\begin{array}{c}\text { CRP } \\
\text { (No \%) }\end{array}$} & Positive & $32(64) \%$ & $0(0) \%$ & \multirow{2}{*}{11.2} & \multirow{2}{*}{$<0.001$} \\
\hline & Negative & $18(36 \%)$ & $30(100 \%)$ & & \\
\hline \multirow{2}{*}{ Blood Culture (No \%) } & Positive & $26(52) \%$ & Not done & \multirow{2}{*}{10.3} & \multirow{2}{*}{$<0.001$} \\
\hline & Negative & $24(48) \%$ & Not done & & \\
\hline
\end{tabular}

$\mathrm{CRP}=\mathrm{C}$ reactive protein

hsCRP $=$ highly sensitive $\mathrm{C}$ reactive protein

Table 3 Comparison between serum NGAL and hs-CRP levels in subgroups of cases (With and without acute kidney injury)

\begin{tabular}{|c|c|c|c|}
\hline & $\begin{array}{c}\text { AKI subgroup } \\
(\mathbf{n = 2 7})\end{array}$ & $\begin{array}{c}\text { No - AKI subgroup } \\
(\mathbf{n}=\mathbf{2 3})\end{array}$ & P value \\
\hline sNGAL (ng/ml) & $27(100 \%)$ & $1(4 \%)$ & $<0.001$ \\
Median & 180 & 56 & $40-73$ \\
IQ range & $163-225$ & $16(69 \%)$ & $<0.001$ \\
\hline hsCRP (mg/l) (No\%) Median & $27(100 \%)$ & $1.7 \pm 1.3$ & \\
\hline
\end{tabular}




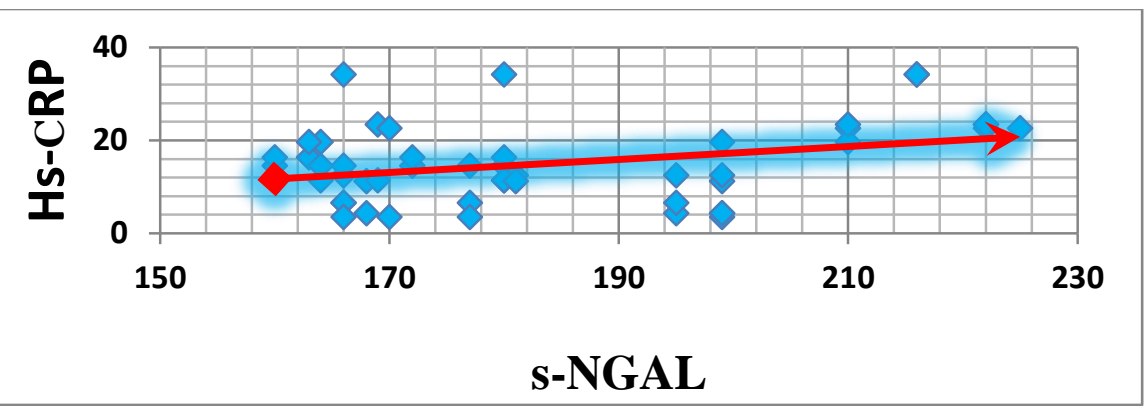

Figure 1 Correlation between serum NGAL and Hs-CRP in septic cases with AKI.

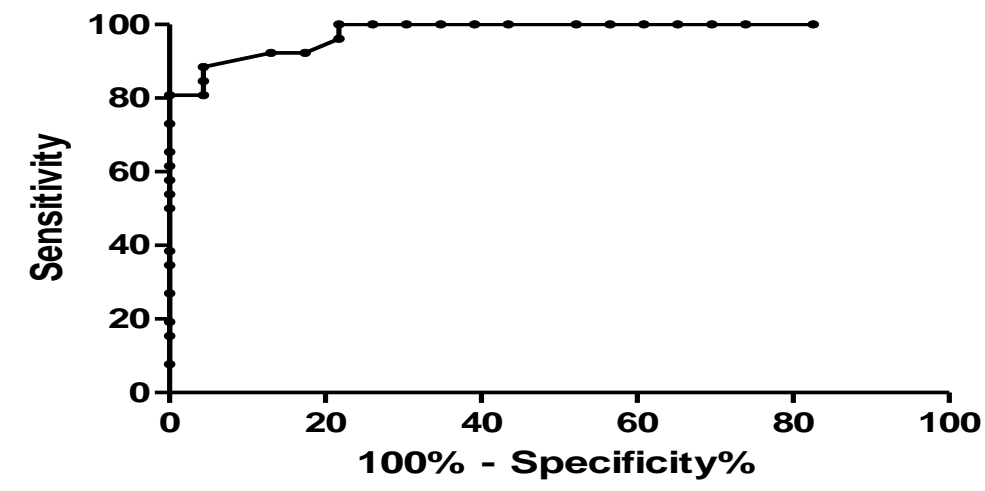

Figure 2 ROC curve between AKI patients \& non-AKI patients as regard Hs-CRP.

ROC curve between AKI patients \& non-AKI patients as regard Hs-CRP
$\begin{array}{ll}\text { Sensitivity } & : 88.5 \% \\ \text { Specificity } & : 95.6 \% \\ \text { Predictive value of +ve result (PPV) } & : 95.26 \% \\ \text { Predictive value of -ve result (NPV) } & : 89.26 \% \\ \text { Cutoff value } & : 3.5\end{array}$

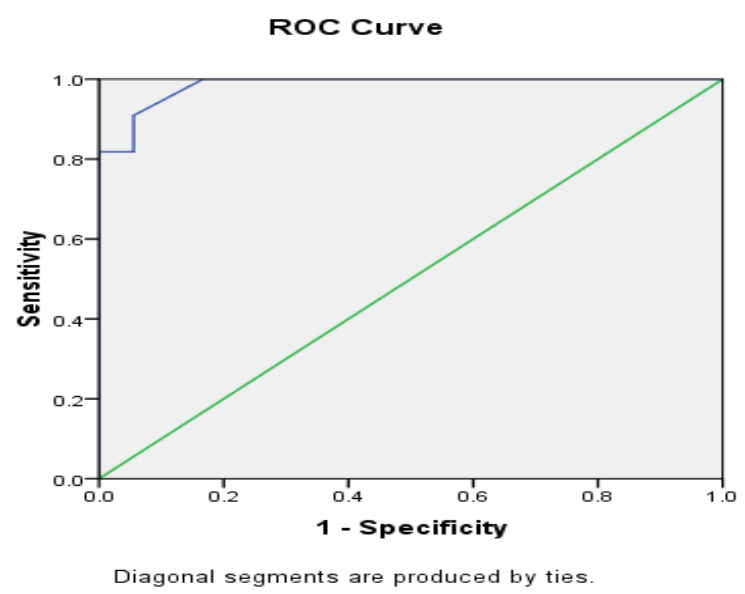

Figure 3 ROC curve for sNGAL as a marker of AKI in septic neonates.

$\underline{\text { ROC curve for } S N G A L}$ as a marker of AKI in septic neonates.:

$\begin{array}{ll}\text { Sensitivity } & : 100 \% \\ \text { Specificity } & : 95.6 \% \\ \text { Accuracy } & : 98 \% \\ \text { Predictive value of +ve result (PPV) } & : 96.4 \% \\ \text { Predictive value of -ve result (NPV) } & : 100 \% \\ \text { Cutoff value } & : 144\end{array}$




\section{Discussion}

Clinical diagnosis of sepsis in newborn infants is difficult because symptoms and signs are non-specific. There is no laboratory test with $100 \%$ specificity and sensitivity. Blood culture has been the gold standard for confirmation of diagnosis but the results are available only after 48-72 hours [6]. In this context, neonatologists and nephrologists have focused their interest on the clinical utility of certain novel AKI biomarkers including Neutrophil Gelatinase-associated Lipocalin (NGAL), a $25-\mathrm{kD}$ protein of the Lipocalin superfamily that is expressed in several tissues including kidney. In humans, serum NGAL is very sensitive, early indicator of AKI in various situations, including sepsis, kidney transplants, and following nephrotoxic drug administration [7].

In this study, serum levels of hs-CRP of (>0.5mg/L) was present in (43) cases $(86 \%)$ which were significantly higher in patients than controls $(\mathrm{p}=0.001)$. This is in agreement with the study done by Abou-Hussein, et al [8] that serum hs-CRP levels in newborns with septicemia were significantly higher than those of control group.

There was a statistically significant association between hsCRP and qualitative CRP levels (100\% of cases with positive qualitative CRP, had positive hs-CRP). Positive CRP cases had positive blood culture results only in $52 \%$ of them. This is in accordance with study done by Boma, et al [9] who reported that $43 \%$ of positive CRP cases had positive blood culture.

Similar, study done by Abou-Hussein, et al [8] in Egypt, and included 51 septic neonates having positive blood culture in the same unit, they found that the sensitivity of hs-CRP in diagnosis of neonatal sepsis was $81.6 \%$, specificity $100 \%$, positive predictive value $100 \%$ and negative predictive value $77 \%$.

In this study, $54 \%$ of cases with sepsis developed acute kidney injury (as defined by serum creatinine level). Serum creatinine was significantly higher in cases group than the control group. The study done by Cruz, et al [10] showed that, in response to renal injury, increases in NGAL levels can predict AKI 24 to $72 \mathrm{~h}$ before diagnostic serum creatinine. Thus, biomarkers that increase as early indicators of acute kidney injury provide the promise of achieving early identification and intervention for those patients. Several studies have found that serum NGAL is a very sensitive and useful biomarker compared with early measurements of serum creatinine in AKI Cruz, et al [10], Shapiro, et al [11].

Levels of serum NGAL (sNGAL) and hs-CRP were significantly higher $(\mathrm{p}<0.001)$ in the -AKI subgroup as compared to no-AKI subgroup. There was a positive correlation between NGAL and hs-CRP in septic cases with AKI( $r=0.33)$. Highly sensitive C-reactive protein might be helpful as a predictive factor of acute kidney injury [3].

In our study, ROC curves analysis suggested that a serum NGAL cutoff value of (144 ng/ml) in neonatal sepsis can predict the development of AKI with sensitivity of $\mathbf{1 0 0} \%$, specificity of $95.6 \%$, and positive predictive value of $96.4 \%$ and negative predictive value of $100 \%$.

Our results were comparable to the results of the study of Cruz, et al [10] which showed that serum NGAL at cut-off values of (> $89.6 \mathrm{ng} / \mathrm{ml})$ had $100 \%$ sensitivity, $92.3 \%$ specificity, to detect acute kidney injury (AKI) in neonatal sepsis.

\section{Conclusion}

Hs-CRP was proved to be very useful biochemical marker for early detection of neonatal sepsis. Also, hs-CRP might be helpful as early predictor of acute kidney injury in septic neonates. Serum NGAL can be considered as a very useful biochemical marker for early detection of AKI in septic neonates.

\section{References}

1. Palevsky PM, Liu KD, Brophy PD, et al. Clinical practice guideline for acute kidney injury. Am J Kidney Dis. 2013; 61(5), 649-672.

2. Yang BH, Acta Neurochir (Wien). Highly sensitive C-reactive protein might be helpful as a predictive factor of acute kidney injury. 2019; 21 (1): 52-59.

3. Stoll BJ. Infections of the Neonatal Infant. Kliegman RM, Behrman RE, Jenson HB and Stanton BF (eds). Nelson Textbook of Pediatrics, 19 editions. Elsevier. 2012; 109: 794-811.

4. Clerico A, Galli C, Fortunato A, et al. Neutrophil gelatinase-associated lipocalin (NGAL) as biomarker of acute kidney injury: a review of the laboratory characteristics and clinical evidences. Clin Chem Lab Med. 2012; 50(9), 1505-1517.

5. Gomella TL. Acute Kidney Injury (AKI) in neonatal sepsis. Indian J.Pharm.Biol.Res. 2015; 51: 939-59.

6. Ashok K, Yadav C, Wilson P. Polymerase Chain Reaction in Rapid Diagnosis of Neonatal Sepsis. Indian Ped. 2015; Vol. 42_JULY 17 p 111-114.

7. Sarafidis K, Tsepkentzi E, Agakidou E, et al. Serum and urine acute kidney injury biomarkers in neonatal sepsis. Pediatr Nephrol. 2012; 27(9):1575-1582.

8. Abou-Hussein HH, El-Khawaga AM, Wahab AA. Creactive protein as a marker for early-onset neonatal sepsis: The good, the better and the best. The Egyptian Journal of Neonatology. 2005; 6 (3): 151-159.

9. Boma I, De1a MD, Remedios F, et al. The diagnostic use of $\mathrm{C}$-reactive protein in the detection of infection among patients with malignancy and connective tissue disease. Phil J.Microbiol Infect Dis. 2012; 30 (3): 108 111.

10. Cruz DN, De Cal M, Garzotto F. Plasma neutrophil gelatinase associated lipocalin is an early biomarker for acute kidney injury in an adult ICU population. Intensive Care Med. 2010; 36(3), 444 - 451.

11. Shapiro NI, Trzeciak S, Hollander JE, et al. The diagnostic accuracy of plasma neutrophil gelatinaseassociatedlipocalin in the prediction of acute kidney injury in emergency department patients with suspected sepsis. Ann Emerg Med. 2010; 56(1), 52-59. 


\section{Statements}

Ethics approval and consent to participate

This study protocol and the consents were approved and deemed sufficient by the Ethical Committee of

Al Azhar University and informed written consent was obtained in every case from their legal guardians.

\section{Consent for publication}

"Not applicable"

Availability of data and material

"Not applicable"

\section{Conflict of interest}

The authors declare no conflict of interest.

\section{Funding}

The authors declare that funding by authors only.

Acknowledgements

Authors thank first and last to God for his great care, support and guidance in every step in our life.

Special thanks to the parents of my patients, for acceptance to participate in this work. 